\title{
Transvenous embolisation via an occluded inferior petrosal sinus for cavernous sinus dural arteriovenous fistulas
}

\author{
Yosuke Kawamura, Tomoji Takigawa, Akio Hyodo, Kensuke Suzuki \\ Department of Neurosurgery, Dokkyo Medical University Saitama Medical Centre, Saitama, Japan
}

\begin{abstract}
Aim of the study: We retrospectively searched for cases of transvenous embolisation for cavernous sinus dural arteriovenous fistulas.
\end{abstract}

Materials and methods: Twenty-five cases underwent transvenous embolisation via the inferior petrosal sinus (IPS). IPS was probed using a standard 0.035 -inch guidewire for microcatheter navigation, which was successful in all cases.

Results: IPS was occluded in 17 cases (68\%). Only one case experienced a complication, where the approach was changed to the contralateral side because of internal jugular vein injury. The relationship between the external auditory canal and the IPS route was reviewed in 18 cases using digital angiography (lateral view). The guidewire passed across, above, or below the external auditory canal in $10(56 \%)$, six (33\%), and two (11\%) cases, respectively.

Clinical implications: No previous reports have analysed the relationship between the external auditory canal and the IPS route. We present a safe and successful technique for approaching the cavernous sinus via the IPS.

Key words: cavernous sinus dural arteriovenous fistulas, occluded inferior petrosal sinus, external auditory canal, guidewire (Neurol Neurochir Pol 2020; 54 (6): 585-588)

\section{Introduction}

A transvenous endovascular procedure is the first-line treatment for cavernous sinus dural arteriovenous fistulas (CSDAVFs) [1]. Accessing the cavernous sinus through an angiographic non-opacified inferior petrosal sinus (IPS) to address carotid cavernous fistulas, and thereby reduce the haemorrhage risk, was first described by Halbach et al. in 1988 [2]. IPS has since become the most common transvenous route regardless of IPS occlusion because it provides a relatively straight and short route to the cavernous sinus [3]. The cavernous sinus can otherwise be accessed via the superior ophthalmic, facial, or superficial middle cerebral vein, or by direct percutaneous puncture $[4,5]$. However, the transvenous approach via the IPS is feasible even when this sinus is thrombosed. Modern hydrophilic microguidewires and microcatheters have improved the efficacy and safety of catheter navigation into the cavernous sinus [6]. Precise location and anatomy of the connection of the remnant of the IPS to the internal jugular vein (IJV) can be determined using 3D venography [7].
While the external auditory canal is an important factor for a successful advance into the cavernous sinus via the IPS, no previous reports have - to the best of our knowledge - documented the relationship between the external auditory canal and the IPS. In addition to researching this in the literature, we retrospectively analysed the success rate of access through the IPS in the treatment of patients who underwent transvenous embolisation for CSDAVFs in our institution.

This report thus provides new technical tips for navigation into the cavernous sinus via the IPS.

\section{Materials and methods}

This descriptive study was approved by our hospital's institutional review board (approval no. 19114). Between December 2009 and December 2019, our institution treated 62 cases of dural arteriovenous fistulas with endovascular surgery; 25 patients underwent transvenous embolisation via the IPS. Angiographic complete occlusion of the IPS was evaluated by reviewing diagnostic cerebral angiography

Address for correspondence: Yosuke Kawamura, Department of Neurosurgery, Dokkyo Medical University Saitama Medical Centre,

2-1-50 Minamikoshigaya, Koshigaya Shi, Saitama 343-8555, Japan, e-mail: yuzufuture1210@yahoo.co.jp 


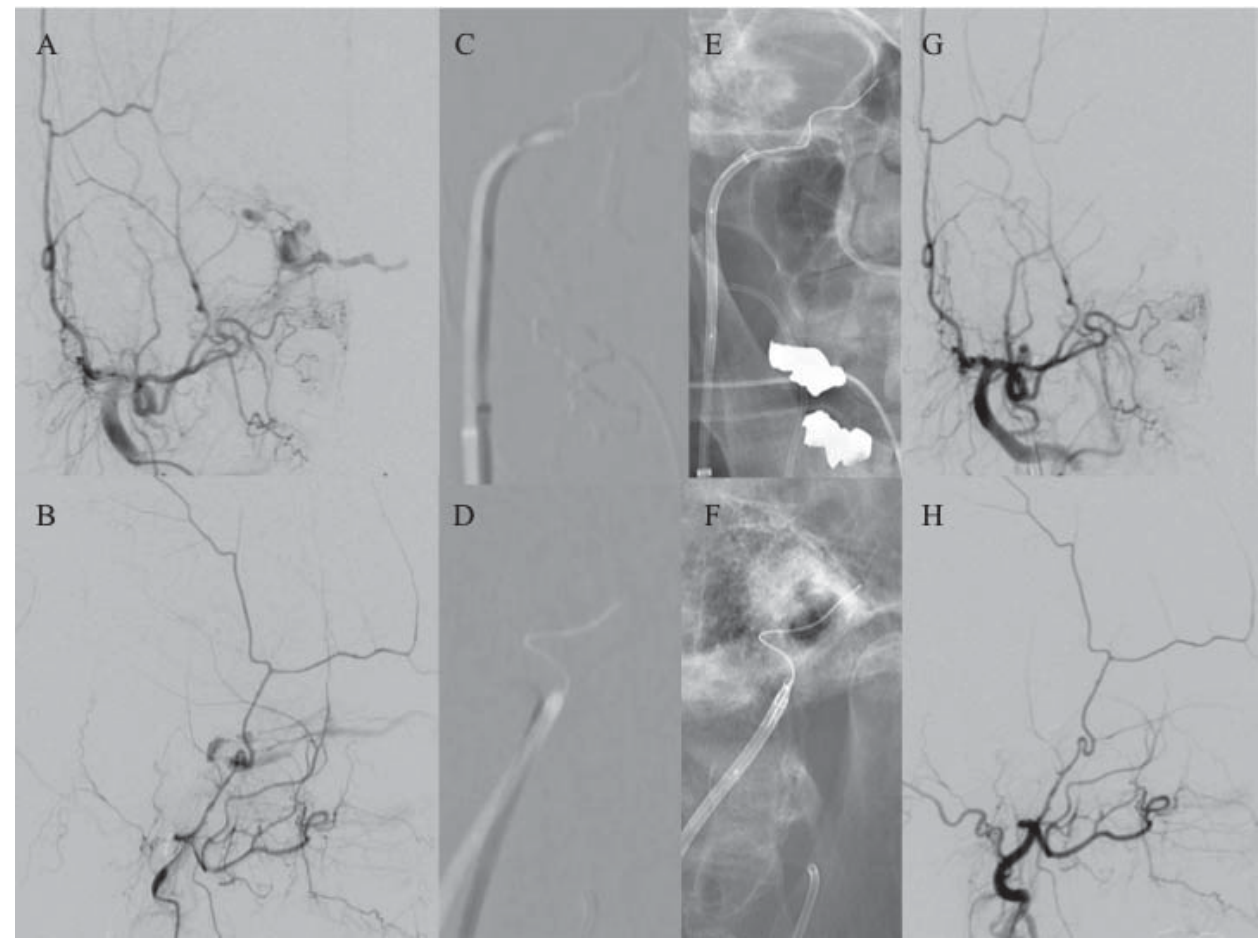

Figure 1. An exemplary case: right external carotid artery angiogram. Fistula is supplied via middle meningeal artery and ascending pharyngeal artery and drains mainly into superior ophthalmic vein and intercavernous sinus (A and B, anteroposterior and lateral views). Inferior petrosal sinus (IPS) is selected and probed with 0.035 -inch guidewire to area of cavernous sinus. White marks are left on road map by pulling back guidewire ( $\mathbf{C}$ and $\mathbf{D}$ ). Microguidewire is introduced into IPS and advanced into cavernous sinus under guidance of roadmap ( $\mathbf{E}$ and $\mathbf{F})$. Transvenous coil and liquid material embolisation is performed; no residual fistula is observed $(\mathbf{G}$ and $\mathbf{H})$

before endovascular treatment to determine whether the IPS on the approach side was visualised as venous drainage of the fistula. The relationship between the external auditory canal and the IPS route was examined using an angiogram lateral view. Seven cases were excluded owing to unclear images and inaccurate angiogram lateral views. Twenty-five patients underwent the following procedure under general anaesthesia: the initial skull position was preoperatively adjusted for accurate anterior-posterior and lateral views. A Flexor Shuttle 7-Fr guiding sheath (Cook Medical, Bloomington, IN, USA) was placed into the IJV. A 6-Fr guiding catheter (Fubuki, Asahi Intecc, Aichi, Japan) was placed into the IPS over a 0.035 -inch outer diameter guidewire (Radifocus, Terumo, Tokyo, Japan). The coaxial system was used for strong supportability. The guidewire was advanced toward the medial superior direction using an anteroposterior view, and the anterior superior direction using a lateral view. When navigation to the cavernous sinus via the IPS using the guidewire proved difficult, a microcatheter (Echelon, Medtronic, Minneapolis, MN, USA) and microguidewire (Chikai, Asahi Intecc, Aichi, Japan) were used. The IPS was probed using a standard 0.035 -inch guidewire. Once the orifice was selected, the guidewire was gently rotated and advanced through the occluded IPS along the imaginary anatomic route. The guidewire was pulled back and removed to leave a bright guidewire track on the roadmap image: a guide for microcatheter navigation through the IPS (Fig. 1). The IPS route was classified into three types defined by the guidewire position: above, across, or below the external auditory canal on the digital angiography lateral view.

\section{Results}

Navigation to the cavernous sinus via IPS was successful in all 25 cases. The IPS was occluded as venous drainage of the fistula on the approach side in 17 cases (68\%). These IPSs were occasionally thrombosed. In one case (4\%), the approach was changed to the contralateral side of the IPS because of injury to the IJV on the approach side. A mean (standard deviation, SD) time of 211.7 (72.7) minutes was needed for the procedure, the mean final absorbed dose was $2,643.8(1,456.1) \mathrm{mGy}$, and the mean volume of the injected contrast medium was 290.9 (233.6) $\mathrm{ml}$. The relationship between the external auditory canal and the IPS route was reviewed in 18 cases using a digital angiography lateral view: the guidewire passed above the external auditory canal in six (33\%, Fig. 2A), across in 10 (56\%, Fig. 2B), and below in two (11\%, Fig. 2C), respectively. 


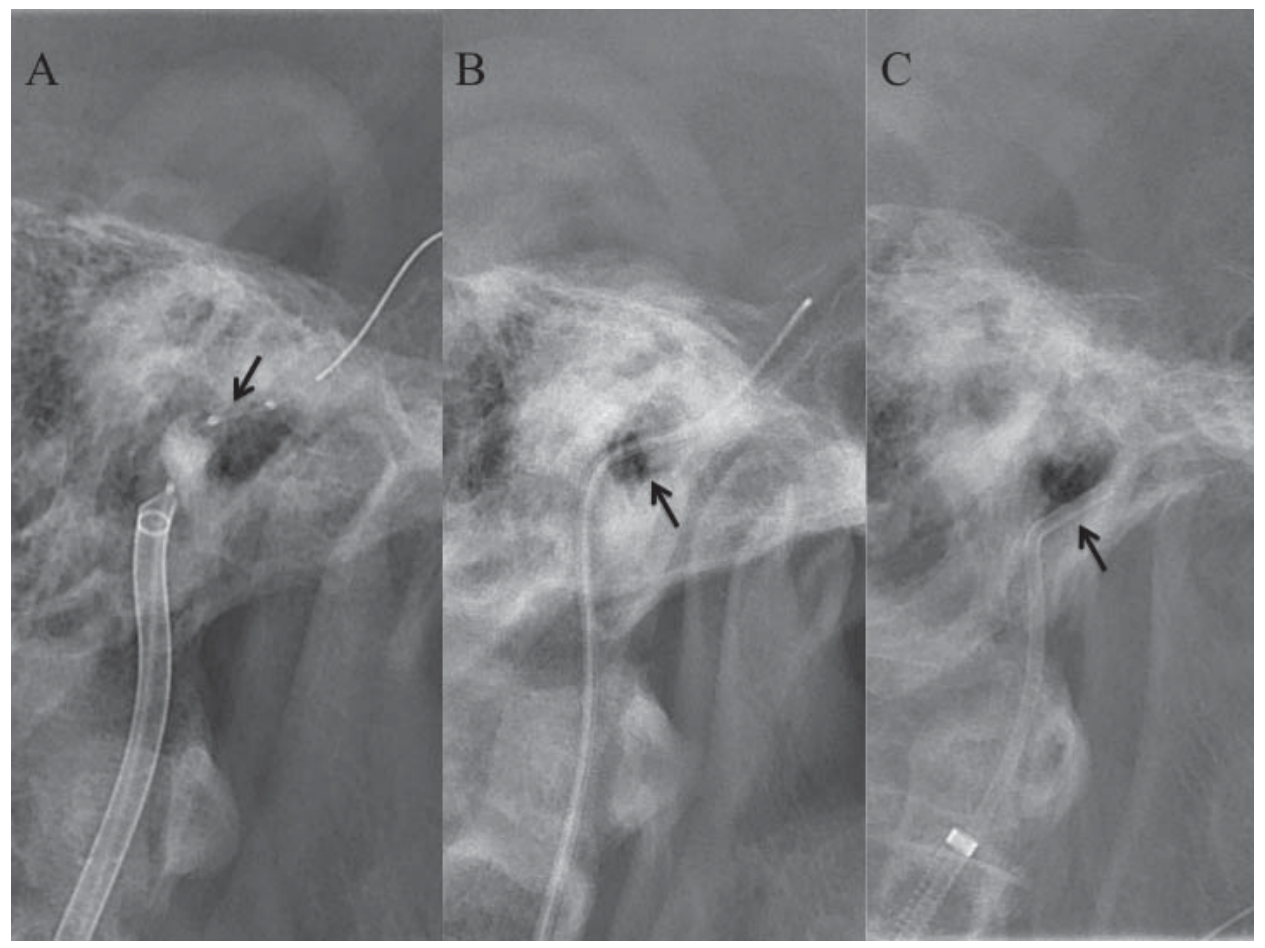

Figure 2. Relationship between external auditory canal and inferior petrosal sinus route: microballoon catheter is passed above external auditory canal (black arrow) in non-subtracted lateral image (A). Microballoon catheter is used to navigate 6-Fr guiding catheter into IPS. Guiding catheter is passed across external auditory canal (black arrow) in non-subtracted lateral image (B). Guiding catheter is passed below external auditory canal (black arrow) in non-subtracted lateral image (C)

\section{Discussion}

Shiu et al. described four distinct types of IPS drainage [8], which were reevaluated by Miller et al [9]. According to this classification, anatomic type IV has no connection between the IJV and IPS. Type IV anatomy is encountered in $1.0 \%$ of patients [10]. When true type IV anatomy is present, the IPS cannot be catheterised via the ipsilateral IJV.

Gailloud et al. reported bilateral extracranial extension of the IPS. The extracranial portion of the IPS was 40 and $38 \mathrm{~mm}$ long on the left and right sides, respectively (measured from the external opening of the jugular foramen to the junction with IJV) [11]. We could not examine the extracranial portion of IPS. Cannulation of a completely occluded IPS is also feasible, especially when using a standard hydrophilic polymer-jacketed 0.035 -inch guidewire as a frontier-wire probing technique. The key technical procedure is probing the orifice of IPS using a guidewire and leaving a bright guidewire track on the roadmap image after pulling back and removing the guidewire. Referring to the roadmap information, a microcatheter over a microguidewire is advanced into the cavernous sinus. The technical success rate is $80.8 \%$ [12]. We probed IPS using a standard guidewire and used the trace on the roadmap image for microcatheter navigation.

Magnetic resonance imaging, using T1 Volumetric Isotropic TSE Acquisition Black Blood and 3D-T1 Fast Field
Echo T1 gadolinium, provides valuable information on the occluded sinus, including the IPS [13]. Our report emphasises the importance of the initial skull position in order to obtain accurate anteroposterior and lateral views and to facilitate access to the cavernous sinus via the IPS. The second most important factor is the guidewire direction. The monitor image must be seen during the procedure to determine whether the guidewire is advanced in the medial superior direction on the anteroposterior view and the anterior superior direction on the lateral view. The method of guidewire use is also important. The Kuru-Kuru method is generally used with a 0.035 -inch guidewire [14]. When advancing into the IPS is difficult, a microcatheter should be used with a microguidewire.

While the external auditory canal is an important consideration when advancing into the cavernous sinus via the IPS, the relationship between the external auditory canal and the IPS route remains unclear. In most of our cases, the guidewire was advanced across the external auditory canal, suggesting that this direction is optimal for a first approach into the cavernous sinus via the IPS.

Thus, navigation to the cavernous sinus via the IPS was successful in all cases, with no complications in $96 \%$ of the first approaches. In most cases, the guidewire advanced across the external auditory canal in the lateral view in digital angiography. The technical analysis could provide some guidance to successfully perform the procedure. 
Acknowledgement: We would like to thank Editage Publication Support for proofreading this article.

Ethics approval: The study design was approved by the institutional review board of Dokkyo Medical University Saitama Medical Centre (approval number 19114).

Conflict of interest: The authors declare no conflict of interest. Funding: This publication was prepared without any external source of funding.

Author contributions: All authors contributed to the study conception and design. Yosuke Kawamura: conceptualisation, data curation, formal analysis, investigation, methodology, software, resources, validation, visualisation, writing original draft. Tomoji Takigawa: supervision, project administration. Akio Hyodo and Kensuke Suzuki: writing - review and editing.

\section{References}

1. Baharvahdat $\mathrm{H}$, Ooi YC, Kim WiJ, et al. Updates in the management of cranial dural arteriovenous fistula. Stroke Vasc Neurol. 2020; 5(1): 50 58, doi: 10.1136/svn-2019-000269, indexed in Pubmed: 32411408.

2. Halbach VV, Higashida RT, Hieshima GB, et al. Transvenous embolization of direct carotid cavernous fistulas. AJNR Am J Neuroradiol. 1988; 9(4): 741-747, indexed in Pubmed: 3135718.

3. Lv X, Wu Z. Anatomic variations of internal jugular vein, inferior petrosal sinus and its confluence pattern: Implications in inferior petrosal sinus catheterization. Interv Neuroradiol. 2015; 21(6): 769-773, doi: 10.1177/1753425915590067, indexed in Pubmed: 26378071.

4. Konstas AA, Song A, Song J, et al. Embolization of a cavernous carotid fistula through the vein of Labbé: a new alternative transvenous access route. BMJ Case Rep. 2017; 2017, doi: 10.1136/bcr-2017013229, indexed in Pubmed: 28963167.

5. Han W, Kim JH, Kang HIn, et al. Transvenous Embolization of Dural Carotid Cavernous Fistula through the Supraorbital Vein. J Cerebrovasc Endovasc Neurosurg. 2019; 21(2): 101-106, doi: 10.7461/ jcen.2019.21.2.101, indexed in Pubmed: 31886146.
6. Benndorf G, Bender A, Lehmann R, et al. Transvenous occlusion of dural cavernous sinus fistulas through the thrombosed inferior petrosal sinus: report of four cases and review of the literature. Surg Neurol. 2000; 54(1): 42-54, doi: 10.1016/s0090-3019(00)00260-3, indexed in Pubmed: 11024506.

7. Srivatanakul $\mathrm{K}$, Osada T, Aoki R, et al. Transvenous embolization of cavernous sinus dural arteriovenous fistula through a thrombosed inferior petrosal sinus utilizing 3D venography. Interv Neuroradiol. 2015; 21(3): 362-365, doi: 10.1177/1591019915581945, indexed in Pubmed: 25977356.

8. Shiu PC, Hanafee WN, Wilson GH, et al. Cavernous sinus venography. Am J Roentgenol Radium Ther Nucl Med. 1968; 104(1): 57-62, doi: 10.2214/ajr.104.1.57, indexed in Pubmed: 5672775.

9. Miller DL, Doppman JL, Chang R. Anatomy of the junction of the inferior petrosal sinus and the internal jugular vein. AJNR Am J Neuroradiol. 1993; 14(5): 1075-1083, indexed in Pubmed: 8237683.

10. Miller DL, Doppman JL. Petrosal sinus sampling: technique and rationale. Radiology. 1991; 178(1): 37-47, doi: 10.1148/radiology.178.1.1845785, indexed in Pubmed: 1845785.

11. Gailloud P, Fasel JH, Muster M, et al. Termination of the inferior petrosal sinus: an anatomical variant. Clin Anat. 1997; 10(2): 92-96, doi: 10.1002/(SICI)1098-2353(1997)10:2<92::AID-CA4>3.0.C0;2-V, indexed in Pubmed: 9058015.

12. Jia ZY, Song YS, Sheen JJ, et al. Cannulation of Occluded Inferior Petrosal Sinuses for the Transvenous Embolization of Cavernous Sinus Dural Arteriovenous Fistulas: Usefulness of a Frontier-Wire Probing Technique. AJNR Am J Neuroradiol. 2018; 39(12): 2301-2306, doi: 10.3174/ajnr.A5868, indexed in Pubmed: 30385474.

13. Hosoo H, Tsuruta W, Nakai Y, et al. The Visualization Methods of Occluded Dural Sinus for Safe Transvenous Embolization of Dural AVFs. World Neurosurg. 2019; 127: e337-e345, doi: 10.1016/j. wneu.2019.02.250, indexed in Pubmed: 30904803.

14. Nemoto S, Mayanagi $\mathrm{Y}$, Kirino T. Cavernous dural arteriovenous fistulas. Transvenous approach and venous drainage of the fistula. Interv Neuroradiol. 1997; 3 Suppl 2: 86-87, doi: 10.1177/15910199970030S216, indexed in Pubmed: 20678392. 